\title{
Optimal Lead Selection for the Study of the $P$ Wave in Sinus Rhythm
}

\author{
A Climent $^{1}$, MS Guillem ${ }^{1}, \mathrm{~J} \mathrm{Millet}^{1}, \mathrm{C} \mathrm{Mora}^{1}$, \\ R Ruiz ${ }^{2}$, FJ Chorro ${ }^{2}$ \\ ${ }^{1}$ UPV, Universidad Politécnica de Valencia, Valencia, Spain \\ ${ }^{2}$ HCUV, Hospital Clínico Universitario de Valencia, Spain
}

\begin{abstract}
Determination of the best electrode positioning for the study of the $P$ wave would lead to a more accurate knowledge of atrial activity. Both Holter recordings and twelve lead electrocardiograms not always show the signal quality enough for a robust $P$ wave detection. New leads could emphasize the $P$ wave and provide more clearly information for the classification of arrhythmias or other atrial disorders. The best lead is defined such as the one presenting the highest amplitude and area in the whole body surface. 64 unipolar surface ECG signals were recorded form active electrodes covering the complete torso surface and 1860 bipolar computed. According their amplitude and area we could be make the conclusion that the best lead positioning for the study of the $P$ wave has been found outside the standard twelve leads. The higher ratios have been found in leads combining recordings from an area close to the right collarbone and the area below left precordial zone.
\end{abstract}

\section{Introduction}

This investigation has carried out with the idea to find a derivation with better features of the $\mathrm{P}$ wave, amplitude in relation to the base line, and the area that this covers.

Find a situation of the torso, where we are able to insure that the atrial signal will be emphasized, will allow improve the classification of different arrhythmias and atrial disorders (for example the paroxistic atrial fibrillation, where the study of $\mathrm{P}$ wave in sinusal functioning is really determining). Besides, in equipments where the number of electrodes is limited, like the Holters, it is necessary make sure that the situation of the derivations will allow detect and classify possible pathologies.

There are several studies about atrial activity recorded. Comparison between $\mathrm{P}$ wave recorded on the standard electrocardiogram [1] and the "Lewis Lead" could show that the derivation II has more possibilities to be the best place to study $\mathrm{P}$ wave. Lewis Lead was presented in the first half of the twentieth century [2] and the convenience or not of the Lewis Lead used is not clear.

Some recent analyses of different possible locations of the 12 derivations on the classical electrocardiograph [3] suggest better locations to take the signals if the objective is work with atrial signal. As well as, some authors have observed that the improvement of some bipolar derivations regarding the classical is significant [4].

Our intention is to define which it is that optimal derivation that should be utilized for the study of the atrial activity. Utilizing techniques of complete study of the electrical cardiac activity on the surface of the torso, better known as body surface potential mapping (BSPM), are possible to find in what unipolar or bipolar derivation has more present the $\mathrm{P}$ wave, exactly our equipment have 64 leads recorders.

\section{Methods \\ Studied patients}

Forty four patients admitted in the HCUV have been involved in this study, as healthy as with different pathologies, where atrial activity was working in sinusal rhythm. Forty one of all them are males and three women.

\section{Equipment}

In order to find those points on the torso, in which by means of an electrocardiograph may appreciate a $\mathrm{P}$ signal with biggest amplitude and area, having an equipment to take simultaneously the bigger number of points on the chest and back was necessary.

The acquisition of the records was made by means the utilization of a system for obtaining body surface potential mapping (BSPM). Which is developed at the Polytechnic University of Valencia [5] and it is based in a commercial system of clinical record 
multichannel with 64 unipolar electrodes: the Active One of the company Biosemi. Specifically, it is equipment with a sampling rate of $2 \mathrm{kHz}$, taking signs with a resolution of 16 bits with $1 \mathrm{uV} / \mathrm{bit}$.

The principal use of these equipments is the realization of potentials maps obtained from the 64 leads. However in our work, more than studying the behaviour of the potential around the torso during the time, it is used the particular results of every leads, and fixing only on the $\mathrm{P}$ wave acquired at each position. Lead configurations used have been showed in the figure 1 .

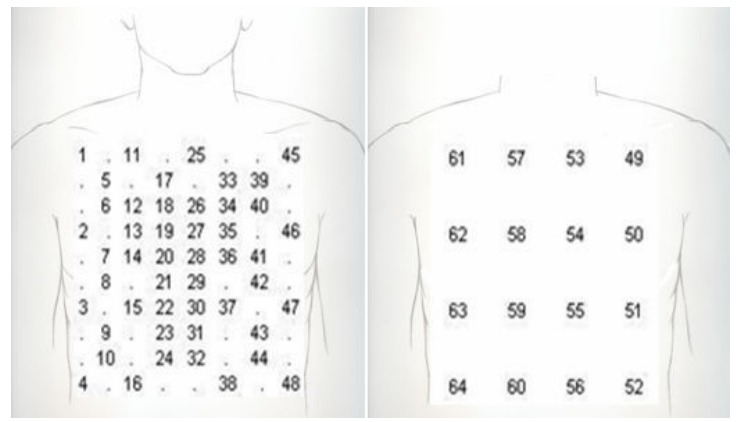

figure 1. Unipolar Lead configurations. Left chest, right back.

With the aim of could do the comparisons between the 12 standard derivations, Lewis's lead, the 64 unipolar leads and all possible bipolar leads (1860 potential distribution), it is necessary place inside this scheme so Lewis's lead like the 12 standard. For it, by means of linear operations, we have recalculated the standard leads from results that our equipment offered us. Specifically, we have taken electrodes that were more near to the standards like our precordial signals. On the other hand the signs that we have taken like more next to the extremities have been 1, 45 and 48. Besides, the Lewis lead must be recorded placing the right arm electrode to the top rights of the manubrium and the left arm electrode to the fifth intercostals space alt the right parasternal line. The difference between the electrodes 13 and 39 has been taken like this Lewis lead.

\section{Procedures and measurements}

The set of signals under study are:

- 64 unipolar online recorded leads

- 12 standard leads

- Lewis Lead

- 1860 bipolar computer leads

Electrical reference, which was fixed during the recording in one electrode was changed. Wilson Central Terminal lead is computed and subtracted to all unipolar leads.
When we have recorded the electrocardiac signals, a preprocessing is applied them. It consist in a high pass filter, with a cutoff frequency of $0.5 \mathrm{~Hz}$ to eliminate great part of the variations on the base line, and, in case of it will be necessary, a $50 \mathrm{~Hz}$ Notch filter.

Bipolar leads have been computed by means of the difference between each and all others points, excepting neighbours, in total we have got 1860 bipolar leads.

To make the analysis, base line must be placed on zero, and then reference all calculations to this value will be able. For this, we took the stretch between $\mathrm{T}$ and $\mathrm{P}$ wave and subtracted the possible offset that could exist, then this stretch is on $0 \mathrm{uV}$ is assured.

After that, for each lead, with at least 10 consecutive pulsations, our algorithm makes a window from each beat. The peaks of every QRS complex are detected using an algorithm based in Tompkins. It derives the ECG to emphasize the peaks, after it squares this derivation and extracts the maximum values found. In order to make the window, the program takes 1024 previous samples and 614 posterior to the QRS's peak, making sure as $\mathrm{P}$ as $\mathrm{T}$ waves are contained in the aforementioned window and besides, every window has the same number of samples.

The algorithm to identify waves is applied to that median signal, so the QRS position will be known. Looking for the inflection points of the signal, crosses with zero on the first derivation, the program found QRSonset and QRSoffset. The algorithm proceeded to defining the $\mathrm{P}$ wave as follow: looking for the peaks found in the beginning of the window, selecting, in case than $\mathrm{P}$ wave exist, the peak of this. After obtaining that significant point, the program looks for Poffset and Ponset checking the slope changes.

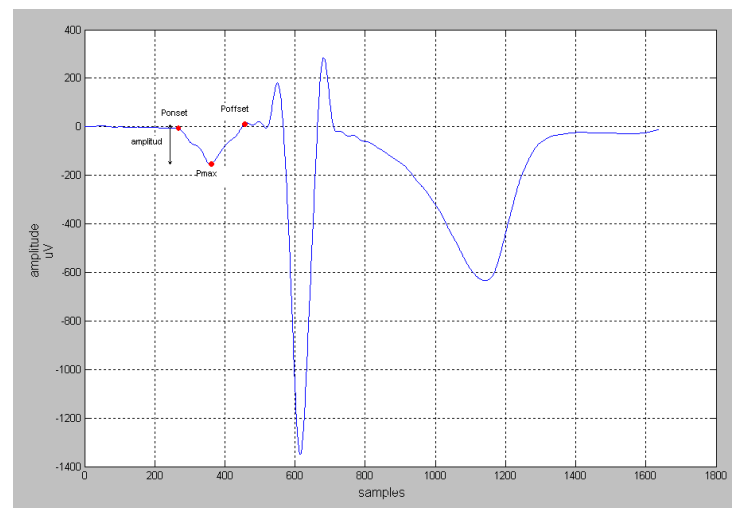

Figure 2. Example of the amplitude measure

On the one hand, amplitude of $\mathrm{P}$ wave is obtained by means of the difference between the aforementioned point $\mathrm{P}$ peak and the base line (figure 2 ). 
On the other hand, the area of the $\mathrm{P}$ wave is assessed from the Ponset to the Poffset: an algorithm of integration, based in the sum of the value of all samples divided by the samples rate, calculates this area (figure 3).

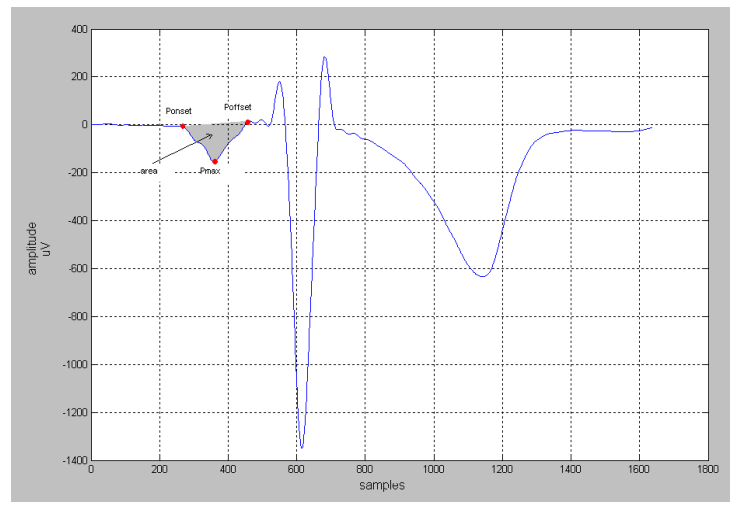

Figure 3. Example of the measure of area

Given that absolute characteristics of the signals, amplitude and area, change between each patients according to their physical characteristics. It is necessary normalize the results to compare them. Specifically, the program looks for the derivation with bigger amplitude in each patient, as well as the derivation with bigger area. They did not have be necessarily the same lead.

After that, the program normalizes the results of one patient dividing the values (amplitude and area) in each derivation by the value of the lead where each parameter was maximum. The results of that operation are two vectors (one for the amplitude and other for the area) of 1930 coefficients (64 unipolar, 180 bipolar and 6 precordials). In this moment, we are already to recognize how many optimum is each lead against to the best in each patient.

Calculating the median of those vectors, all leads will have amplitude and area ratios about how many near from the best derivation are. We will be in a position to obtain the derivations where the atrial activity may be found and studied more robustly.

\section{Results}

With the ratio vectors it is possible represent the quality of each lead and therefore sees the improvement introduced by each new leads. The figures 5 and 6 show the ratios of amplitude and area respectively for better precordial, Lewis Lead and better unipolar and bipolar leads. These last are the ones that clearly have better ratio.

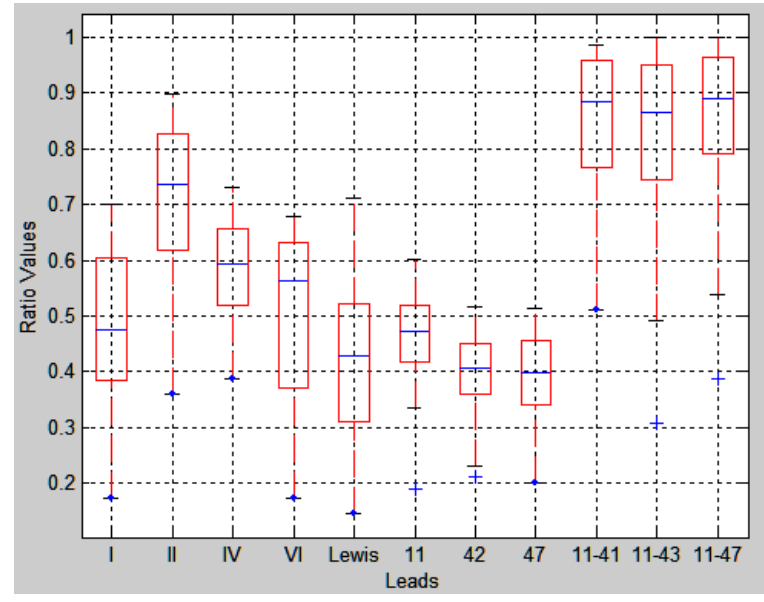

Figure 4. Amplitude results.

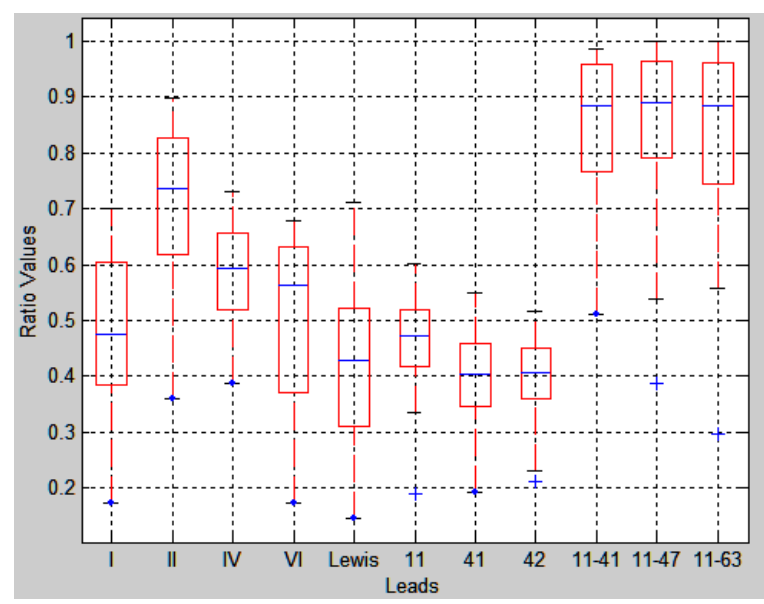

Figure 5. Area results.

In table 1 the absolute values and ratios of the best leads in each type are showed.

\begin{tabular}{|l|l|l|l|l|l|}
\hline & Lead & $\begin{array}{l}\text { Amplitude } \\
\mathrm{uV}\end{array}$ & $\begin{array}{l}\text { Amplitude } \\
\text { Ratio }\end{array}$ & $\begin{array}{l}\text { Area } \\
\mathrm{uV} * \mathrm{sec}\end{array}$ & Area Ratio \\
\hline Standard & II & $-50 \pm 30$ & $0.71 \pm 0.14$ & $30 \pm 12$ & $0.70 \pm 0.14$ \\
\hline Bipolar & $11-47$ & $-167 \pm 50$ & $0.84 \pm 0.15$ & $100 \pm 35$ & $0.84 \pm 0.14$ \\
\hline Unipolar & 11 & $-90 \pm 30$ & $0.46 \pm 0.10$ & $45 \pm 17$ & $0.46 \pm 0.08$ \\
\hline Lewis & $13-39$ & $80 \pm 50$ & $0.41 \pm 0.15$ & $44 \pm 18$ & $0.42 \pm 0.16$ \\
\hline
\end{tabular}

Table 1. Best particular results.

\section{Discussion and conclusions}

As in the cases studied by Jon E. Madies [1], lead II is the one that obtain better results. Its ratio is considerably similar to the best lead in a bigger number of cases. The concrete value of the ratio obtained for amplitude is $0.73 \pm 0.1$ (figure 4) and for area $0.71 \pm 0.1$ (figure 5), that means its value is in average $70 \%$ of the measured for the best lead. There are really 
promising results if we compare them with the remaining classical leads, which strangely exceed a $50 \%$. About Lewis lead, we have could verify that it show appreciably inferiors values, around $40 \%$.

The unipolar leads results never are better than $50 \%$. All the same, in figures 5 and 6 are showed results of leads 11, ,42 and 47 of amplitude and 11, 41 and 42 of area, because these are unipolar electrodes with better ratios.

About new bipolar leads there are measures even worse than the results obtained by the unipolar points. However some of those combined signals are better than classical leads. The values obtained for leads combining recordings from an area close to the right collarbone and the area below left precordial zone are close to the $80 \%$ for each one of the patients. That position was suggested by Robert L. Lux [3], and now with this study, it can be proved like the appropriate lead.

A first conclusion is that the Lewis lead is not the best possibility to detect the $\mathrm{P}$ wave. That lead has results around $40 \%$ than in area as in amplitude.

Inside the standard lead, derivation II is the optimal for the study of the atrial activity. Anyone of the other standard leads has interesting results. Perhaps, these would get an overview of the heart functioning, however they are not the best possibility to appreciate in particular the atrial activity.

The positions of the unipolar leads with better results are placed one close to the right collarbone, and the other below left precordial zone (figure 6). Regions in which the polarity of the $\mathrm{P}$ wave was inverted one in relation to the other; on the one hand, close to the right collarbone the peak of the $\mathrm{P}$ wave is negative, whereas in the left abdomen the signal measured is positive.

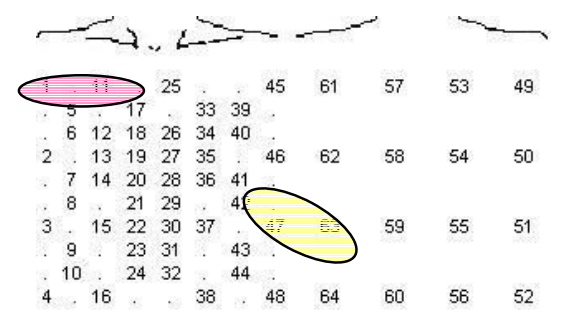

Figure 6. Areas with better unipolar leads.

After this appreciation it is logical think that the relation between signals measured at both areas will give the bipolar leads with better ratios. In fact, the maximum ratios are obtained in the measures 11-47 and 11-63, higher than $80 \%$. Then, it is possible sure that these new bipolar leads will be better than standard to study atrial activity, at least in sinus rhythm.

The utility of these conclusions can be interesting in the study of pathologies referent to the atrial activity, especially in long Holter recorders, when $\mathrm{P}$ wave detection is more critical.

\section{Acknowledgements}

Give any acknowledgements here.

\section{References}

[1] Madias, JE. Comparison of $\mathrm{P}$ waves recorded on the standard electrocardiogram, the "Lewis lead," and "salinefilled central venous catheter"-based intracardiac electrocardiogram. The American Journal of Cardiology Vol. 94, Issue 4 , August 2004, Pages 474-478

[2] Constant J. Learning Electrocardiography. (3rd Ed ed.), Little Brown and Co, Boston (1987).

[3] Waktare JE, Gallagher MM, Murtagh A, Camm AJ, Malik M. Optimum lead positioning for recording bipolar atrial electrocardiograms during sinus rhythm and atrial fibrillation. Clin Cardiol. 1998 Nov;21(11):825-30.

[4] Robert L. Lux, University of Utah, Salt Lake City, and UT, USA, Richard Greg, AARC, Philips Medical Systems, Andover, MA, USA. New Leads for P wave Detection and Arrhytmia Classification Journal of Electrocardiology Vol.37 Sup. 2004

[5] Guillem, M. S., Millet, J., Bodí, V., Mora, C., and Chorro, F. J. Integration of a Body Surface Potential Mapping System. X Mediterranean Conference on Medical and Biological Engineering. 2004.

Address for correspondence

Andreu Martínez Climent

Departamento de Ingeniería Electrónica, ETSIT,

Universidad Politécnica de Valencia,

46022 Valencia, Spain

bamarcli@teleco.upv.es 